\title{
A Deep Neural Network Model for the Prediction of Major Adverse Cardiovascular Event Occurrences in Patients with non-ST-Elevation Myocardial Infarction
}

\author{
Huilin ZHENG ${ }^{\text {a }}$, Syed Waseem Abbas SHERAZI ${ }^{\text {a }}$, Sang Hyeok SON ${ }^{\text {a }}$, and Jong Yun \\ $\mathrm{LEE}^{\mathrm{a}, 1}$ \\ ${ }^{a}$ Department of Computer Science, Chungbuk National University, Cheongju, \\ Chungbuk 28644, South Korea
}

\begin{abstract}
Cardiovascular disease (CVD) is one of the major causes of death all over the world and the mortality rate is higher than other causes. Hence, we propose a novel deep neural network (DNN)-based prediction model for the major adverse cardiovascular event (MACE) occurrences in patients with non-ST-Elevation myocardial infarction (NSTEMI) to improve the prediction accuracy of CVD. The research contents are described as follows. First, for the experiment, we use the Korean Acute Myocardial Infarction Registry (KAMIR-NIH) dataset with 2 years follow-ups and then preprocess the extracted data, such as processing the missing values, solving the imbalance problem, and applying the normalization meth to scale all the datasets in the same range for the experiment. Then we design a DNN-based prognosis model for the occurrences of MACE in NSTEMI patients. Finally, we evaluate the proposed model's performance and compare it with several applied machine learning algorithms, such as logistic regression, K-Nearest Neighbors, decision tree, and support vector machine. The result shows that the performance of our proposed method outperformed other machine learning-based prediction models.
\end{abstract}

Keywords. Cardiovascular disease, major adverse cardiovascular event, deep learning, NSTEMI, imbalanced data

\section{Introduction}

Cardiovascular disease (CVD) has been one of the major problems for all human beings and is considered the highest cause of mortality globally. There was an approximated 17.9 million death from CVD and accounted for $31 \%$ of all worldwide deaths in 2016 [1]. In addition, the CVD death is also severe in South Korea [2]. The treatment of the patients who suffering the CVD is expensive in hospitals. Therefore, the prediction of CVD at the early step is necessary for all people, especially for the aged, since the CVD burden will rise a lot with population aging [3]. Traditional prediction of the CVD has concentrated on regression-based models like Global Registry of Acute Coronary Events

${ }^{1}$ Corresponding Author: Jong Yun Lee, Department of Computer Science, Chungbuk National University, Cheongju, Chungbuk 28644, South Korea; E-mail: jongyun@chungbuk.ac.kr. 
(GRACE) [4], Framingham risk scores [5], Thrombolysis in Myocardial Infarction (TIMI) [6], etc. Even though those regression-based models are commonly accepted tools for CVD, they were developed long since and cannot address the complex interactions between the risk factors $[7,8,9]$.

Recently, various machine learning (ML) techniques have been developed for the prediction of CVD, which can reveal the complex interactions between different risk factors and also improve the accuracy of the prognosis model [7-10]. A ML-based prognosis model for the 1-year mortality was applied in Korean acute coronary syndrome patients using several ML algorithms such as generalized linear model (GLM), gradient boosting machine, etc., which showed that the ML-based prediction models enhanced the performances than the traditional regression-based GRACE model [7]. In addition, a paper used ML-based methods such as GLM, decision tree models, and Bayes models to predict the mortality of in-hospital for Chinese patients with ST-Elevation myocardial infarction (STEMI), which achieved higher prognosis performance than the previous inhospital mortality prognosis STEMI models [10]. On the other hand, as a set of ML methods, the deep learning (DL) techniques also have shown strong abilities in many domains recently because of the increase of computational power [11]. In a paper, the authors developed a DL-based AI algorithm to predict the mortality of acute heart failure patients [12]. Another paper also generated a DL-based prognosis model of the occurrences of major adverse cardiovascular event (MACE) during the 1, 6, and 12month follow-ups after discharge in patients with acute myocardial infarction using a deep neural network (DNN), where the result showed that the DL-based prediction model was superior to the GRACE model [13]. Nevertheless, most of the previous studies, which have used the conventional regression or ML-based models with limited risk factors, could not predict CVD accurately.

Hence, we propose a novel AI-based prognosis model using the DNN to get more accurate performance for the prognosis of MACE occurrences in patients with non-STElevation myocardial infarction (NSTEMI). The research contents are shown as follows. Firstly, we extract the experimental NSTEMI patients' data from the raw KAMIR-NIH dataset and preprocess it by dealing with the missing values, solving the imbalance problem, and using the normalization approach to scale all the experimental data in the same range for the experiment. Secondly, we develop our DNN-based prognosis model for the MACE occurrences in NSTEMI patients. Finally, we evaluate and compare the performance of the proposed model with several popular machine learning algorithms, such as logistic regression (LR), K-Nearest Neighbors (KNN), decision tree (DT), and support vector machine (SVM).

\section{Materials and Methods}

The overall experimental framework of this paper mainly includes three parts, which is shown in Figure 1. The first part is the data extraction and preprocessing of the raw KAMIR-NIH dataset, which extracts and preprocesses the dataset for the experiment. The second part is the model generation, where we design the proposed DNN-based model and apply the other ML-based prognosis models for the occurrences of MACE in NSTEMI patients. The final part is the performance evaluation, where the performances of the proposed DNN-based and other ML-based prognosis models are evaluated and compared by several useful performance measures. 


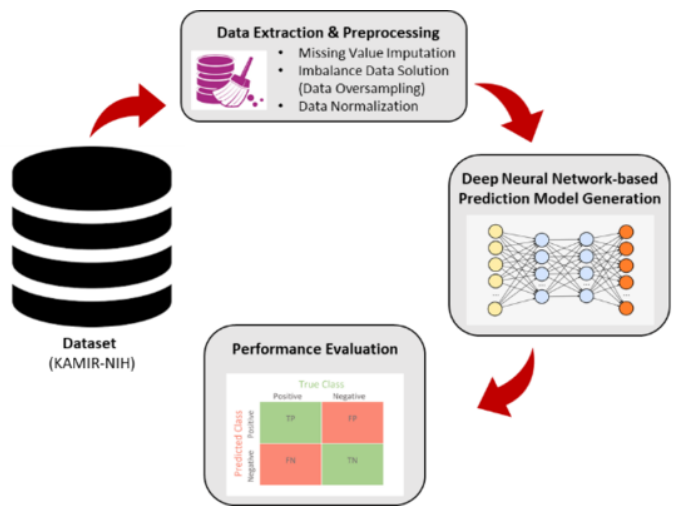

Figure 1. The overall experimental framework.

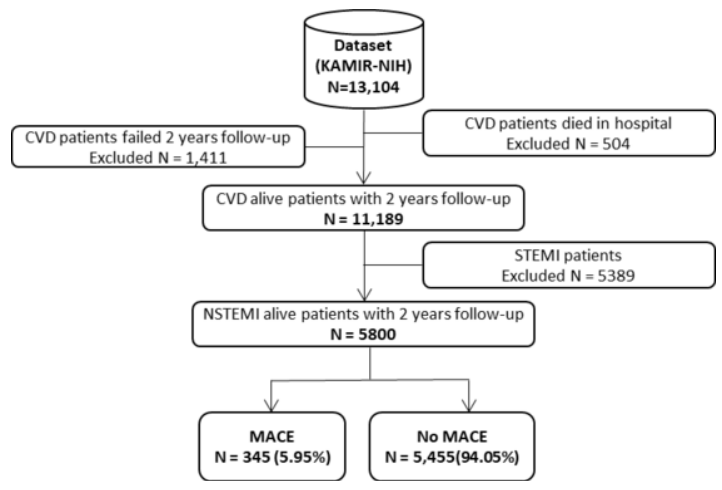

Figure 2. The process of data extraction.

\subsection{Data source and preprocessing}

The KAMIR-NIH dataset is used for the experiment in this paper. It is the first nationwide, multi-center registry with CVD patients in South Korea [14]. The dataset extraction process for getting the experimental dataset is shown in Figure 2, which includes 5,800 NSTEMI alive patients with 2 years follow-ups. The extracted variables include total 60 numerical and nominal features, where the nominal features are gender, previous chest pain, chest pain, electrocardiogram (ECG) use at admission, Killip class, dyspnea, ST-change on ECG, previous angina pectoris, previous myocardial infarction (MI), previous cerebrovascular disease, previous heart failure, diabetes mellitus, hypertension, dyslipidemia, family history of early age ischemic heart disease, family history of heart disease, history of smoking, symptoms of MI, pre-TIMI flow of target vessel, post-TIMI flow of target vessel, percutaneous coronary intervention (PCI), MI ECG change, use of coronary angiogram (CAG), result of $\mathrm{CAG}$, use of thrombolysis, outcome of thrombolysis, initial diagnosis of STEMI \& NSTEMI, final diagnosis of STEMI \& NSTEMI, coronary artery bypass grafting (CABG), use of ECG in hospital, discharge type of patient, MACE, and the numerical features comprise age, diastolic blood pressure (DBP), Systolic Blood Pressure (SBP), height, weight, heart rate, abdominal circumference, white blood cells (WBC), WBC lymphocyte, WBC neutrophil, platelet, hemoglobin, creatinine, glucose, total cholesterol, maximum creatine kinase MB, 
maximum creatine kinase peek, TnI, low-density lipoprotein cholesterol, high-density lipoprotein cholesterol, hsCRP, HbA1c, BNP, NTproBNP, triglyceride, discharge heart rate, discharge DBP, discharge SBP. The target variable MACE is defined as myocardial infarction (MI), CABG, non-cardiac death, cardiac death, and re-percutaneous coronary intervention (re-PCI), whereas other events are considered as No_MACE. Nevertheless, there are numerous missing values in the extracted data. Therefore, we impute those missing values with zero in this paper to solve the missing values. In addition, the data imbalanced problem is obvious in our experimental data, where the ratio of the No_MACE and MACE is 15.8:1. Many different techniques were implemented to deal with the data imbalance issue, such as data sampling, cost-sensitive techniques, etc. [15]. This paper use one of the data sampling methods to overcome the imbalance issue for the experimental data, which is called borderline synthetic minority oversampling (Borderline-SMOTE1) [16]. It is an extension of the Synthetic Minority Oversampling Technique (SMOTE) technique [17], which can only produce synthetic minority cases to oversample the minority group along the decision boundary between the two classes [16]. After that, we apply the MinMaxScaler to normalize the training dataset into range 0 to 1 , and then use it to transform the test dataset for improving the prediction accuracy. The mathematical equation of the MinMaxScaler is shown in Eq. (1) [18].

$$
x_{\text {scaled }}=\frac{x-x_{\min }}{x_{\max }-x_{\min }}
$$

\subsection{Proposed method}

A DNN is an artificial neural network with manifold hidden layers between the input and output layers [19]. In this paper, a DNN-based prognosis model was proposed which is presented in Figure 3. The proposed DNN-based prediction model includes two hidden layers. To get optimal performance, the neurons of each hidden layer are tuned by the grid search method because the grid search is simple to implement and parallelization is trivial [20], where there are 100 optimal neurons in each hidden layer. In modern artificial neural networks, the activation function is an important feature that decides whether a neuron should be activated or not. Many different types of typical activation functions like tanh, sigmoid, rectified linear unit (ReLU), etc. were introduced [21]. In this paper, the ReLU activation function is used by reason of its efficiency. It outputs the input directly if it is a positive value or simply replaces the input with zero if it is a negative value [21]. In addition, the Adam optimizer is used to update network weights iterative which is an extension to stochastic gradient descent-based optimizer and computationally efficient [22]. And during the model training, the dropout layer [23] (dropout rate $=0.2$ ) and early stopping [24] methods are used to reduce overfitting and improve the generation of DNN.

We have compared the designed DNN-based prediction model with four popular ML-based prognosis models for MACE occurrences, such as LR [25], KNN [26], DT [27], and SVM [28], where the LR algorithm uses a sigmoid function to map the predicted values to probabilities within the range of $[0,1]$ and then assign examples to a discrete set of classes; KNN algorithm captures the idea of similarity and put the new case into the category that is most similar to the available categories; DT algorithm learns simple decision rules using the tree representation to solve the classification or regression problems; SVM algorithm creates a hyperplane that can segregate a higher dimensional space into classes. The default parameters of the four ML algorithms in the scikit-learn 
package are used to create the compared models, where the max_iter parameters of LR and SVM are set to 20,000 for the solvers to converge.

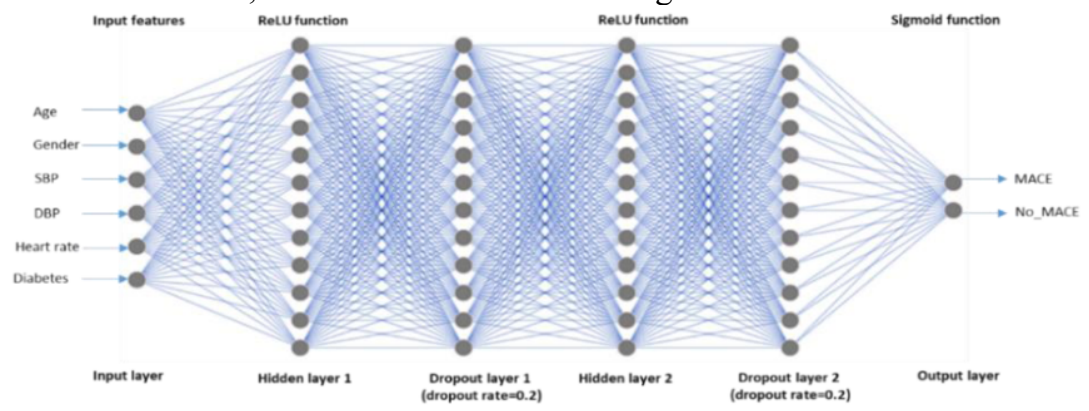

Figure 3. Proposed deep neural network-based prediction model for MACE occurrences.

\subsection{Performance measures}

For the evaluation of our prediction model, we split our dataset as $70 \%$ training and $30 \%$ test dataset, where the training dataset is applied to create the proposed prognosis model for the occurrences of MACE in NSTEMI patients, and the test dataset is utilized to evaluate the performance of the proposed prediction model. The performance is evaluated by the precision, recall, F1-score, accuracy, and the area under the ROC curve (AUC) for the applied prediction models.

\section{Results and Discussion}

In the experiment, we proposed a DNN-based model for the prognosis of MACE occurrences in patients with NSTEMI. The performance of the proposed prediction model was compared with four popular ML-based prognosis models such as LR, KNN, DT, and SVM. The results of the performance comparison for the proposed and compared prognosis models are shown in Table 1, where the best results are presented in bold. The Receiver Operating Characteristic (ROC) curves of the proposed and compared prediction models are shown in Figure 4.

The result shows that the performance of the proposed prognosis model outperformed other statistic-based, instance-based, and tree-based prediction models with the highest accuracy, precision, F1-score, and AUC scores as 0.9594, 0.9487, 0.9611 , and 0.9589 , respectively, however, the KNN-based prediction model got the best recall as 0.9864 . In addition, in the four compared prediction models, the DT and KNNbased prediction models got higher performance with more than $91 \%$ accuracy than the LR and SVM-based models.

Table 1. Results of the performance comparison of the proposed and compared prediction models.

\begin{tabular}{c|c|c|c|c|c}
\hline & LR & SVM & KNN & DT & Proposed Model \\
\hline Accuracy & 0.7372 & 0.7372 & 0.9132 & 0.9248 & $\mathbf{0 . 9 5 9 4}$ \\
Precision & 0.7576 & 0.7612 & 0.8646 & 0.9197 & $\mathbf{0 . 9 4 8 7}$ \\
Recall & 0.7217 & 0.7152 & $\mathbf{0 . 9 8 6 4}$ & 0.9361 & 0.9739 \\
F1-score & 0.7392 & 0.7375 & 0.9215 & 0.9278 & $\mathbf{0 . 9 6 1 1}$ \\
AUC & 0.7378 & 0.738 & 0.9108 & 0.9245 & $\mathbf{0 . 9 5 8 9}$ \\
\hline
\end{tabular}




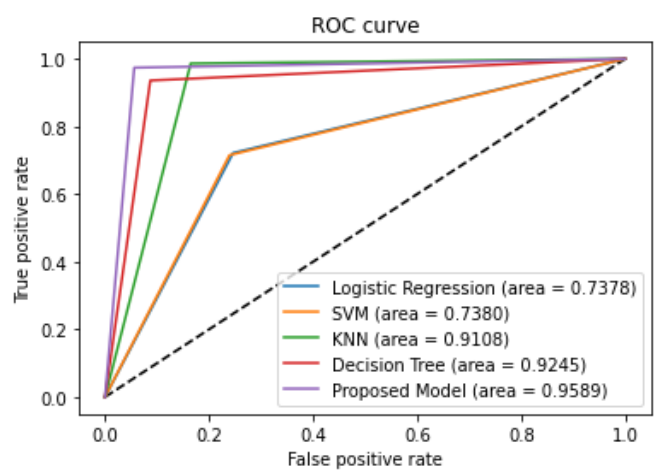

Figure 4. Receiver Operating Characteristic (ROC) curves of the proposed and compared prediction models.

\section{Conclusion}

In this paper, a DNN-based model was proposed for the prediction of MACE occurrences in NSTEMI patients using the KAMIR-NIH dataset with 2 years follow-ups. The performance of the designed DNN-based prognosis model was evaluated and compared with the other four ML-based models, which showed that the proposed DNN-based prognosis model achieved the greatest performance around 96\% accuracy to the prognosis of MACE occurrences in patients with NSTEMI. Consequently, the DL method showed more accurate prediction results for MACE occurrences in NSTEMI patients.

However, there are several limitations to this paper. First, the result of this paper is difficult to apply to other populations since we experimented on the Korean dataset. Second, we only applied one type of data sampling technique to oversample the minority class without comparing with other popular methods to solve the data imbalance problem.

\section{Acknowledgments}

This work was supported in part by the Korea Institute for Advancement of Technology (KIAT) Grant through the Korean Government by the Ministry of Trade, Industry and Energy (MOTIE) under Grant N0002429, in part by the Basic Science Research Program through the National Research Foundation of Korea (NRF) by the Ministry of Education under Grant 2017R1D1A1A02018718, and in part by the Ministry of Science and ICT (MSIT), South Korea, through the Grand Information Technology Research Center Support Program supervised by the Institute for Information and Communications Technology Planning and Evaluation (IITP) under Grant IITP-2021-2020-0-01462.

\section{References}

[1] World Health Organization, Cardiovascular diseases (CVDs). Available from: https://www.who.int/newsroom/fact-sheets/detail/cardiovascular-diseases-(cvds) [Accessed 06 Sept 2021]

[2] Shin HY, Lee JY, Song J, Lee S, Lee J, Lim B, ... Huh S. Cause-of-death statistics in the Republic of Korea, 2014. Journal of the Korean Medical Association. 2016;59(3):221-232. 
[3] Roth GA, Mensah GA, Johnson CO, Addolorato G, Ammirati E, Baddour LM, ... Fuster V. Global Burden of Cardiovascular Diseases Writing Group. Global burden of cardiovascular diseases and risk factors, 1990-2019: update from the GBD 2019 paper. Journal of the American College of Cardiology. 2020;76(25):2982-3021.

[4] Fox KA, Dabbous OH, Goldberg RJ, Pieper KS, Eagle KA, Van de Werf F, ... Granger CB. Prediction of risk of death and myocardial infarction in the six months after presentation with acute coronary syndrome: prospective multinational observational paper (GRACE). bmj. 2006;333(7578):1091.

[5] Wilson PW, D’Agostino RB, Levy D, Belanger AM, Silbershatz H, Kannel WB. Prediction of coronary heart disease using risk factor categories. Circulation. 1998;97(18), 1837-1847.

[6] Antman EM, Cohen M, Bernink PJ, McCabe CH, Horacek T, Papuchis G, ... Braunwald E. The TIMI risk score for unstable angina/non-ST elevation MI: a method for prognostication and therapeutic decision making. Jama. 2000;284(7):835-842.

[7] Sherazi SWA, Jeong YJ, Jae MH, Bae JW, Lee JY. A machine learning-based 1-year mortality prediction model after hospital discharge for clinical patients with acute coronary syndrome. Health informatics journal. 2020;26(2):1289-1304.

[8] Patel B, Sengupta P. Machine learning for predicting cardiac events: what does the future hold?. Expert review of cardiovascular therapy. 2020;18(2):77-84.

[9] Zheng H, Sherazi SWA, Lee JY. A Stacking Ensemble Prediction Model for the Occurrences of Major Adverse Cardiovascular Events in Patients With Acute Coronary Syndrome on Imbalanced Data. IEEE Access. 2021;9:113692-113704.

[10] Li X, Liu H, Yang J, Xie G, Xu M, Yang Y. Using machine learning models to predict in-hospital mortality for ST-elevation myocardial infarction patients. In MEDINFO 2017: Precision Healthcare through Informatics. IOS Press, 2017;476-480.

[11] Bizopoulos P, Koutsouris D. Deep learning in cardiology. IEEE reviews in biomedical engineering. 2018;12:168-193.

[12] Kwon JM, Kim KH, Jeon KH, Lee SE, Lee HY, Cho HJ, ... Oh BH. Artificial intelligence algorithm for predicting mortality of patients with acute heart failure. PloS one. 2019;14(7):e0219302.

[13] Kim YJ, Saqlian M, Lee JY. Deep learning-based prediction model of occurrences of major adverse cardiac events during 1-year follow-up after hospital discharge in patients with AMI using knowledge mining. Personal and Ubiquitous Computing. 2019;1-9.

[14] Korea Acute Myocardial Infarction Registry. Available from: http://kamir5.kamir.or.kr [Accessed 06 Sept 2021]

[15] Ali A, Shamsuddin SM, Ralescu AL. Classification with class imbalance problem. Int. J. Advance Soft Compu. Appl. 2013;5(3).

[16] Han H, Wang WY, Mao BH. Borderline-SMOTE: a new over-sampling method in imbalanced data sets learning. In International conference on intelligent computing. Hefei, China, 2005;878-887. Springer, Berlin, Heidelberg.

[17] Chawla NV, Bowyer KW, Hall LO, Kegelmeyer WP. SMOTE: synthetic minority over-sampling technique. Journal of artificial intelligence research. 2002;16:321-357.

[18] Bisong E. Introduction to Scikit-learn. In Building Machine Learning and Deep Learning Models on Google Cloud Platform. Apress, Berkeley, CA; 2019. 215-229.

[19] Sze V, Chen YH, Yang TJ, Emer JS. Efficient processing of deep neural networks: A tutorial and survey. Proceedings of the IEEE. 2017;105(12):2295-2329.

[20] Bergstra J, Bengio Y. Random search for hyper-parameter optimization. Journal of machine learning research. 2012;13(2):281-305.

[21] Sharma S, Sharma S. Activation functions in neural networks. Towards Data Science. 2017;6(12):310316.

[22] Kingma DP, Ba J. Adam: A method for stochastic optimization. arXiv preprint arXiv:1412.6980. 2014;1-15.

[23] Srivastava N, Hinton G, Krizhevsky A, Sutskever I, Salakhutdinov R. Dropout: a simple way to prevent neural networks from overfitting. The journal of machine learning research. 2014;15(1):1929-1958.

[24] Prechelt L. Early stopping-but when?. In Neural Networks: Tricks of the trade. 1998;55-69. Springer, Berlin, Heidelberg.

[25] Bagley SC, White H, Golomb BA. Logistic regression in the medical literature: Standards for use and reporting, with particular attention to one medical domain. Journal of clinical epidemiology, 2001;54(10):979-985.

[26] Cover T, Hart P. Nearest neighbor pattern classification. IEEE transactions on information theory. 1967;13(1):21-27.

[27] Breiman L, Friedman JH, Olshen RA, Stone CJ. Classification and Regression Trees. Routledge, 1984.

[28] Hearst MA, Dumais ST, Osman E, Platt J, Scholkopf B. Support vector machines. IEEE Intell. Syst. Appl. 2008;13(4):18-28. 